\title{
Body mass index and its dose-response relationship with the incidence and risk of tuberculosis, a population-based cohort study
}

\author{
Jinou Chen \\ Shun Zha \\ Yunnan Centers for Disease Control and Prevention \\ Jinglong Hou \\ Yunnan Center for Disease Control and Prevention \\ Kunyun Lu \\ Yunnan Center for Disease Control and Prevention \\ Yubing Qiu \\ Yunnan Center for Disease Control and Prevention \\ Rui Yang \\ Yunnan Center for Disease Control and Prevention \\ Ling Li \\ Yunnan Center for Disease Control and Prevention \\ Yunbin Yang \\ Yunnan Center for Disease Control and Prevention \\ Lin Xu ( $\nabla$ xulinth@hotmail.com)
}

Yunnan Center for Disease Control and Prevention https://orcid.org/0000-0001-6074-2178

\section{Research article}

Keywords: Tuberculosis, Body index mass, Dose-response relationship

Posted Date: May 12th, 2020

DOl: https://doi.org/10.21203/rs.3.rs-26565/v1

License: (c) (i) This work is licensed under a Creative Commons Attribution 4.0 International License. Read Full License 


\section{Abstract \\ Background}

Many pieces of evidence presented the body mass index (BMI) was inversely associated with tuberculosis (TB). BMI was radically changed during the past decades in China. The aim of the study was to evaluate the contemporary relationship between BMI and tuberculosis incident.

\section{Methods}

A population-based prospective cohort included 26022 community participants was conducted. Three rounds of tuberculosis screening were implemented between June 2013 and December 2015. The main exposure was defined as baseline BMI, and was categorized into 3 levels: underweight (<18.5 kg/m²), normal ( 18.5 to $\left.24.0 \mathrm{~kg} / \mathrm{m}^{2}\right)$, overweight or obese $\left(\geq 24.0 \mathrm{~kg} / \mathrm{m}^{2}\right)$. The active tuberculosis incident in the second or third round screening was the study outcome. The dose-response relationship between BMI and tuberculosis incidence as well as tuberculosis risk were analyzed.

\section{Results}

During the followed up of 2.25 years, 43 cases developed tuberculosis in 44574.4 person-years. The log-linear dose-response relationship between BMI and tuberculosis incidence was fitted (adjusted $R^{2}=0.95$ ). In multivariable Cox proportional regression, overweight and obese was associated with a lower risk of incident tuberculosis compared with normal weight (adjusted hazard ratio [aHR], 0.34; $95 \%$ confidence interval [Cl] $0.14-0.82, p<0.01$ ), the inverse dose-response association between $\mathrm{BMI}$ and tuberculosis risk was characterized by restricted cubic spline. In subgroups analysis, the risk of tuberculosis reduced $78 \%$ in overweight or obese (aHR, 0.22; 95\% Cl 0.05-0.97, $p=0.05)$ and $64 \%(\mathrm{aHR}, 0.36 ; 95 \% \mathrm{Cl} 0.12-1.00, p=0.05)$ compared with normal weight among female and elderly.

\section{Conclusion}

Our study revealed that high BMI was a protective factor in tuberculosis development. Precise dose-response relationship between BMI and the incident tuberculosis, as well as the risk of tuberculosis progression in contemporary Chinese adulthood, will benefit to disease control policy.

\section{Background}

Tuberculosis (TB) is an airborne communicable disease that leads to a high burden of public health [1]. About $9 \%$ of the entire world newly diagnosed pulmonary tuberculosis (PTB) were from high burden countries of China, therefore gave an estimated incidence of 61 (95\% Cl: 52-70) pre 100 000 population as the country contributed $20 \%$ of the global population [2].

Body mass index (BMI) was an efficient, convenient, and measurable indicator for body characteristics in the population. The association between BMI and tuberculosis incidence was studied before. In 2010, a systemic review included 6 remote cohort studies presented homogeneous inverse relationships between $\mathrm{BMI}$ and tuberculosis incidence in the BMI range of $18.5-30 \mathrm{~kg} / \mathrm{m}^{2}$ under different settings and tuberculosis burden [3].

Over past decades, China had dramatic growth in the economy, accompanied by the demographic and epidemiological profile shift, disease spectrum, and risks have changed simultaneously. The prevalence of obesity in the year of 2014 was 61 or 27 times higher than it in 1975 among Chinese male or female adult [4]. The percentage changes in agestandardised summary exposure values (SEVs) were ranked the first for high BMI as a risk factor from 1990 to 2017 [5]. This dramatic change for the main exposure of BMI might have a nonnegligible impact on the outcome of the tuberculosis incident. Contemporary association for the BMI after adjusting the multiple demographic and epidemiological factors linked to the major outcome of tuberculosis incident are not established. Besides, the precise dose-response relationship between $\mathrm{BMI}$ and tuberculosis incidence as well as disease progress risk remains unclear in a high burden setting.

To address this knowledge gap, the present study aims to evaluate the relationship between BMI and incidence of tuberculosis based on a populationbased tuberculosis screening cohort from 2013 to 2015 in China, yet to examine the dose-response relationship of BMI and tuberculosis risks, overall and in subgroups.

\section{Methods}

\section{Data source and study population}

The present cohort study was implemented between June 2013 and December 2015. The study site was Dongchuan county of Yunnan province in the southwestern mountainous region of China. It was a complex, multistage sampling prospective tuberculosis screening cohort among communities, the detailed study design has been documented elsewhere [6]. In general, three rounds of tuberculosis screening were implemented in the study time frame among randomly sampled communities. The cohort baseline investigation was conducted in 2013 , home visits, and face-to-face surveys were carried out by trained community health workers (CHWs). Of the 35691 community residents visited by CHWs, 2271 refused to participate in the study, or temporary 
resident was not enrolled in the study. The cohort enrolled 33386 eligible participants, standardized questionnaires comprised of suspected tuberculosis symptoms, demographic, comorbidity, or epidemiological exposure and lifestyle were applied for baseline data collection. These participants' age under 15 years $(n=7364)$ or these diagnosed active tuberculosis in baseline screening $(n=34)$ were excluded. Finally, the present research included 26022 participants for analysis (Fig. 1). The participants in the fixed cohort were prospectively followed up until the occurrence of tuberculosis, death, moved out of the study field, or to December 2015. The second and third round of tuberculosis screening was implemented in each year of 2014 and 2015 , by applying the same algorithm with the baseline survey.

\section{Measurement of BMI and covariates}

BMI records in the baseline survey were the main exposure to this study. Individual BMI was calculated as the ratio of the weight in kilograms as numerator divided by the square of height in meters as the denominator. We categorized the continuous BMl into three groups: underweight $\left(<18.5 \mathrm{~kg} / \mathrm{m}^{2}\right)$, normal $\left(18.5\right.$ to $\left.24.0 \mathrm{~kg} / \mathrm{m}^{2}\right)$, overweight or obese $\left(\geq 24.0 \mathrm{~kg} / \mathrm{m}^{2}\right)$ according to criteria of weight for adults ruled by Ministry of Health of the People's Republic of China in 2013 [7].

Most of the participants' characteristics were self-reported when interviewed by CHWs at the baseline investigation. Covariates included demographic, comorbidity, medical history, and lifestyle information: sex (male, female), age (< 65 years, $\geq 65$ years old), ethnicity (Han, other minority), education level (illiterate or semi-illiterate, primary school, secondary school, college and above, unknown), marital status (married, single, widowed/divorced/separated/other), family annual income per capital (< the median of $7200 \mathrm{RMB}, \geq$ the median of $7200 \mathrm{RMB}$ ), close contact to an index TB case (yes, no), chronic bronchitis (yes, no), pneumoconiosis (yes, no), smoking (never, former, current)and drinking habit (never, former, current), Bacillus Calmette-Guerin (BCG) vaccine scar in the arm (yes, no) were collected. Information of previously treated tuberculosis cases (yes, no) were examined by matching with the case report in Tuberculosis Information Management System (TBIMS), known human immunodeficiency virus or acquired immune deficiency syndrome (HIV/AIDS) cases (yes, no) were checked by local Center for Disease Control and Prevention (CDC) database. Known diabetes mellitus (DM) cases (yes, no) were matched to personal health records of the National Project of Basic Public Health Service (BPHS) for conformation.

\section{Assessment of tuberculosis}

The incident of tuberculosis and the time-to-incident was defined as the study outcome, those with followed up to December 2015 , death, moved out of the study field, or refused to participate in second or third round screening were defined as censored data.

Tuberculosis diagnostic algorithm was: after home visit by CHWs, those tuberculosis symptoms positivity was defined as suspect, combined with those age over 65 years, close contact to an index case, previously treated tuberculosis, known DM and known HIV/AIDS cases underwent chest X-ray (CXR) and health examination. After that, those with abnormal radiography get further sputum smear test, three sputum samples (one at spot sputum, one at night and one on the next morning) were requested for laboratory diagnosis. Patients with a smear acid-fast bacilli positivity were diagnosed as laboratoryconfirmed tuberculosis. Those abnormal radiographic with negative smear, their CXRs were reviewed by the diagnostic committee and were diagnosed as clinically diagnosed tuberculosis.

\section{Statistical analysis}

We compared the proportions in BMI groups by the chi-square test. We computed the person-years and incident rate (IR) of tuberculosis in all participants, by $\mathrm{BMI}$ level and other covariates. Incident rate ratio (IRR) and 95\% $\mathrm{Cl}$ were calculated to compare incidence between groups.

We applied the Kaplan-Meier analysis and Log-Rank test to compare the cumulative hazard of time to tuberculosis incidents in different BMI levels. Denary logarithm transferred tuberculosis incidence was regressed with the average BMI in three levels by the linear regression model, the determination coefficient of $R^{2}$ and adjusted $R^{2}$ were used to evaluate the log-linear model. Cox proportional hazard regression models were fitted to estimate the hazard ratio (HR) of variables with the corresponding $95 \% \mathrm{Cl}$, BMl categorization of normal weight was set as the reference level. We adjusted for covariates in multivariate analysis, the bidirectional stepwise variable selection strategy was applied, the parameter for the criterion of inclusion was $p$-value $\leq 0.05$, and exclusion was $p$-value $\geq 0.1$. After variable selection, we adjusted the categorical factors to control confounder: sex, age, ethnicity, marital status, previously treated tuberculosis, smoking status, and alcohol use. The linear trend test for BMI was performed by replacing BMI as an ordinal variable includes in the multivariate model. DM might correlate with both BMI and tuberculosis, we corrected DM in a different Cox regression model.

We examined the dose-response association between BMI and predicted linear cumulative hazard of tuberculosis for the Cox regression model adjusted for age, sex, ethnicity, marital status, previously treated TB, smoking status, alcohol use, known DM, and continuous BMI, overall and in risk subgroups. The relationship was evaluated with restricted cubic spline regression (RCS), the spline knots were the quartiles (25\%, 50\%, and $75 \%$ ) of continuous BMI.

Subgroup analysis for gender and age were applied after adjusting the covariates. The interaction effect between BMI and gender or age was examined. The effect was defined as an added term of cross-product the BMI level and gender or age in the multivariable Cox regression model after controlling other confounders.

All statistical analysis was performed by R software 3.5.2 (http://www.Rproject.org). The level of $p<0.05$ was set as statistical significance. 
Table 1

The baseline characteristics of the cohort categorized by body mass index.

\begin{tabular}{|c|c|c|c|c|c|}
\hline \multirow[t]{4}{*}{ Characteristics } & \multicolumn{5}{|c|}{ BMI category, kg/m² } \\
\hline & \multirow{2}{*}{$\begin{array}{l}\text { Underweight } \\
<18.5\end{array}$} & \multirow{2}{*}{$\begin{array}{l}\text { Normal } \\
18.5 \text { to } 24.0\end{array}$} & \multirow{2}{*}{$\begin{array}{l}\text { Overweight and } \\
\text { obese } \\
\geq 24.0\end{array}$} & \multirow[t]{2}{*}{ Overall } & \multirow[t]{2}{*}{$\begin{array}{l}\mathrm{p}- \\
\text { value }\end{array}$} \\
\hline & & & & & \\
\hline & $\mathrm{n}(\%)$ & $\mathrm{n}(\%)$ & $\mathrm{n}(\%)$ & $\mathrm{n}(\%)$ & \\
\hline Total & $2042(7.9 \%)$ & $\begin{array}{l}16009 \\
(61.5 \%)\end{array}$ & $7971(30.6 \%)$ & $\begin{array}{l}26022 \\
(100.0 \%)\end{array}$ & \\
\hline Sex & & & & & $<0.01$ \\
\hline Male & $838(41.0 \%)$ & $\begin{array}{l}7245 \\
(45.3 \%)\end{array}$ & $4313(54.1 \%)$ & $\begin{array}{l}12396 \\
(47.6 \%)\end{array}$ & \\
\hline Female & $\begin{array}{l}1204 \\
(59.0 \%)\end{array}$ & $\begin{array}{l}8764 \\
(54.7 \%)\end{array}$ & $3658(45.9 \%)$ & $\begin{array}{l}13626 \\
(52.4 \%)\end{array}$ & \\
\hline Age (years) & & & & & $<0.01$ \\
\hline Mean (SD) & $38.9(21.4)$ & $44.4(17.1)$ & $48.5(14.7)$ & $45.2(17.0)$ & \\
\hline Median & 34 & 43 & 48 & 44 & \\
\hline$<65$ & $\begin{array}{l}1693 \\
(82.9 \%)\end{array}$ & $\begin{array}{l}13771 \\
(86.0 \%)\end{array}$ & $6765(84.9 \%)$ & $\begin{array}{l}22229 \\
(85.4 \%)\end{array}$ & \\
\hline$\geq 65$ & $349(17.1 \%)$ & $\begin{array}{l}2238 \\
(14.0 \%)\end{array}$ & $1206(15.1 \%)$ & $3793(14.6 \%)$ & \\
\hline \multicolumn{6}{|l|}{ Ethnicity } \\
\hline Han & $\begin{array}{l}1867 \\
(91.4 \%)\end{array}$ & $\begin{array}{l}14804 \\
(92.5 \%)\end{array}$ & $7436(93.3 \%)$ & $\begin{array}{l}24107 \\
(92.6 \%)\end{array}$ & $<0.01$ \\
\hline Other minority & $175(8.6 \%)$ & $1205(7.5 \%)$ & $535(6.7 \%)$ & 1915 (7.4\%) & \\
\hline \multicolumn{6}{|l|}{ Education level } \\
\hline Illiterate or semi-illiterate & $155(7.6 \%)$ & $1190(7.4 \%)$ & $703(8.8 \%)$ & $2048(7.9 \%)$ & $<0.01$ \\
\hline Primary school & $292(14.3 \%)$ & $\begin{array}{l}2749 \\
(17.2 \%)\end{array}$ & $1630(20.4 \%)$ & $4671(18.0 \%)$ & \\
\hline Secondary school & $\begin{array}{l}1249 \\
(61.2 \%)\end{array}$ & $\begin{array}{l}9432 \\
(58.9 \%)\end{array}$ & $4445(55.8 \%)$ & $\begin{array}{l}15126 \\
(58.1 \%)\end{array}$ & \\
\hline College and above & $339(16.6 \%)$ & $\begin{array}{l}2603 \\
(16.3 \%)\end{array}$ & $1181(14.8 \%)$ & $4123(15.8 \%)$ & \\
\hline Unknown & $7(0.3 \%)$ & $35(0.2 \%)$ & $12(0.2 \%)$ & $54(0.2 \%)$ & \\
\hline \multicolumn{6}{|l|}{ Marital status } \\
\hline Married & $\begin{array}{l}1086 \\
(53.2 \%)\end{array}$ & $\begin{array}{l}11638 \\
(72.7 \%)\end{array}$ & $6538(82.0 \%)$ & $\begin{array}{l}19262 \\
(74.0 \%)\end{array}$ & $<0.01$ \\
\hline Single & $785(38.4 \%)$ & $\begin{array}{l}2759 \\
(17.2 \%)\end{array}$ & $635(8.0 \%)$ & $4179(16.1 \%)$ & \\
\hline Widowed/divorced/separated/other & $171(8.4 \%)$ & $\begin{array}{l}1612 \\
(10.1 \%)\end{array}$ & $798(10.0 \%)$ & $2581(9.9 \%)$ & \\
\hline \multicolumn{6}{|c|}{$\begin{array}{l}\text { Family annual income per capita (Median }=7200 \\
\text { RMB) }\end{array}$} \\
\hline Below the median & $\begin{array}{l}1072 \\
(52.5 \%)\end{array}$ & $\begin{array}{l}8061 \\
(50.4 \%)\end{array}$ & $3842(48.2 \%)$ & $\begin{array}{l}12975 \\
(49.9 \%)\end{array}$ & $<0.01$ \\
\hline Above the median & $970(47.5 \%)$ & $\begin{array}{l}7948 \\
(49.6 \%)\end{array}$ & $4129(51.8 \%)$ & $\begin{array}{l}13047 \\
(50.1 \%)\end{array}$ & \\
\hline \multicolumn{6}{|l|}{ Previously treated TB } \\
\hline No & $\begin{array}{l}2019 \\
(98.9 \%)\end{array}$ & $\begin{array}{l}15929 \\
(99.5 \%)\end{array}$ & $7936(99.6 \%)$ & $\begin{array}{l}25884 \\
(99.5 \%)\end{array}$ & $<0.01$ \\
\hline
\end{tabular}

Abbreviations: BMI, Body mass index; SD, Standard deviation; HIV, Human immunodeficiency virus; AIDS, Acquired immune deficiency syndrome; BCG, Bacillus Calmette-Guerin vaccine 


\begin{tabular}{|c|c|c|c|c|c|}
\hline \multirow[t]{4}{*}{ Characteristics } & \multicolumn{5}{|c|}{ BMI category, $\mathrm{kg} / \mathrm{m}^{2}$} \\
\hline & & & $\begin{array}{l}\text { Overweight and } \\
\text { obese }\end{array}$ & Overall & $\begin{array}{l}\text { p- } \\
\text { value }\end{array}$ \\
\hline & $<18.5$ & 18.5 to 24.0 & $\geq 24.0$ & & \\
\hline & $\mathrm{n}(\%)$ & n (\%) & $\mathrm{n}(\%)$ & $\mathrm{n}(\%)$ & \\
\hline Yes & $23(1.1 \%)$ & $80(0.5 \%)$ & $35(0.4 \%)$ & $138(0.5 \%)$ & \\
\hline \multicolumn{6}{|l|}{ Close contacts } \\
\hline No & $\begin{array}{l}2039 \\
(99.9 \%)\end{array}$ & $\begin{array}{l}15990 \\
(99.9 \%)\end{array}$ & 7960 (99.9\%) & $\begin{array}{l}25989 \\
(99.9 \%)\end{array}$ & $<0.01$ \\
\hline Yes & $3(0.1 \%)$ & $19(0.1 \%)$ & $11(0.1 \%)$ & $33(0.1 \%)$ & \\
\hline \multicolumn{6}{|l|}{ Known HIV/AIDS } \\
\hline No & $\begin{array}{l}2040 \\
(99.9 \%)\end{array}$ & $\begin{array}{l}15995 \\
(99.9 \%)\end{array}$ & 7966 (99.9\%) & $\begin{array}{l}26001 \\
(99.9 \%)\end{array}$ & 0.82 \\
\hline Yes & $2(0.1 \%)$ & $14(0.1 \%)$ & $5(0.1 \%)$ & $21(0.1 \%)$ & \\
\hline \multicolumn{6}{|l|}{ Known diabetes } \\
\hline No & $\begin{array}{l}2021 \\
(99.0 \%)\end{array}$ & $\begin{array}{l}15693 \\
(98.0 \%)\end{array}$ & 7669 (96.2\%) & $\begin{array}{l}25383 \\
(97.5 \%)\end{array}$ & 0.68 \\
\hline Yes & $21(1.0 \%)$ & $316(2.0 \%)$ & $302(3.8 \%)$ & $639(2.5 \%)$ & \\
\hline \multicolumn{6}{|l|}{ Chronic bronchitis } \\
\hline No & $\begin{array}{l}1938 \\
(94.9 \%)\end{array}$ & $\begin{array}{l}15334 \\
(95.8 \%)\end{array}$ & 7565 (94.9\%) & $\begin{array}{l}24837 \\
(95.4 \%)\end{array}$ & $<0.01$ \\
\hline Yes & $88(4.3 \%)$ & $480(3.0 \%)$ & $351(4.4 \%)$ & $919(3.5 \%)$ & \\
\hline Unknown & $16(0.8 \%)$ & $195(1.2 \%)$ & $55(0.7 \%)$ & $266(1.0 \%)$ & \\
\hline Pneumoconiosis & & & & & $<0.01$ \\
\hline No & $\begin{array}{l}2032 \\
(99.5 \%)\end{array}$ & $\begin{array}{l}15978 \\
(99.8 \%)\end{array}$ & 7945 (99.7\%) & $\begin{array}{l}25955 \\
(99.7 \%)\end{array}$ & \\
\hline Yes & $10(0.5 \%)$ & $31(0.2 \%)$ & $26(0.3 \%)$ & $67(0.3 \%)$ & \\
\hline \multicolumn{6}{|l|}{ Smoking status } \\
\hline Never & $\begin{array}{l}1689 \\
(82.7 \%)\end{array}$ & $\begin{array}{l}12178 \\
(76.1 \%)\end{array}$ & $5441(68.3 \%)$ & $\begin{array}{l}19308 \\
(74.2 \%)\end{array}$ & 0.02 \\
\hline Former & $57(2.8 \%)$ & $485(3.0 \%)$ & $280(3.5 \%)$ & $822(3.2 \%)$ & \\
\hline Current & $296(14.5 \%)$ & $\begin{array}{l}3346 \\
(20.9 \%)\end{array}$ & $2250(28.2 \%)$ & $5892(22.6 \%)$ & \\
\hline Alcohol use & & & & & $<0.01$ \\
\hline Never & $\begin{array}{l}1778 \\
(87.1 \%)\end{array}$ & $\begin{array}{l}12884 \\
(80.5 \%)\end{array}$ & $5870(73.6 \%)$ & $\begin{array}{l}20532 \\
(78.9 \%)\end{array}$ & \\
\hline Former & $76(3.7 \%)$ & $594(3.7 \%)$ & $396(5.0 \%)$ & $1066(4.1 \%)$ & \\
\hline Current & $188(9.2 \%)$ & $\begin{array}{l}2531 \\
(15.8 \%)\end{array}$ & 1705 (21.4\%) & $4424(17.0 \%)$ & \\
\hline BCG scar & & & & & $<0.01$ \\
\hline Yes & $\begin{array}{l}1265 \\
(61.9 \%)\end{array}$ & $\begin{array}{l}9466 \\
(59.1 \%)\end{array}$ & $4570(57.3 \%)$ & $\begin{array}{l}15301 \\
(58.8 \%)\end{array}$ & \\
\hline No & $660(32.3 \%)$ & $\begin{array}{l}5571 \\
(34.8 \%)\end{array}$ & $2896(36.3 \%)$ & 9127 (35.1\%) & \\
\hline Unknown & 117 (5.7\%) & 972 (6.1\%) & $505(6.3 \%)$ & 1594 (6.1\%) & \\
\hline
\end{tabular}


Table 2

Follow up and the tuberculosis incident by associated factors in the cohort.

\begin{tabular}{|c|c|c|c|c|c|c|c|c|}
\hline \multirow[t]{2}{*}{ Characteristics } & \multirow{2}{*}{$\begin{array}{l}\text { Number of } \\
\text { participants } \\
\text { in cohort } \\
\text { n (\%) }\end{array}$} & \multirow[t]{2}{*}{$\begin{array}{l}\text { Person- } \\
\text { years }\end{array}$} & \multirow{2}{*}{$\begin{array}{l}\text { Number } \\
\text { of } \\
\text { cases }\end{array}$} & \multicolumn{2}{|c|}{$\begin{array}{l}\text { Incident rate } \\
\text { (per } 100 \text { 000) }\end{array}$} & \multicolumn{2}{|c|}{$\begin{array}{l}\text { Incident rate } \\
\text { ratio }\end{array}$} & \multirow[t]{2}{*}{$p$-value } \\
\hline & & & & \multicolumn{2}{|l|}{$(95 \% \mathrm{Cl})$} & \multicolumn{2}{|c|}{$(95 \% \mathrm{Cl})$} & \\
\hline \multicolumn{9}{|l|}{ All } \\
\hline & 26022 & 44574.4 & 43 & 96.5 & $\begin{array}{l}(69.8- \\
129.9)\end{array}$ & & & \\
\hline \multicolumn{9}{|l|}{ Sex } \\
\hline Male & $\begin{array}{l}12396 \\
(47.6 \%)\end{array}$ & 21231.7 & 26 & 122.5 & $\begin{array}{l}(80.0- \\
179.4)\end{array}$ & ref & & \\
\hline Female & $\begin{array}{l}13626 \\
(52.4 \%)\end{array}$ & 23342.7 & 17 & 72.8 & $\begin{array}{l}(42.4- \\
116.6)\end{array}$ & 0.59 & $\begin{array}{l}(0.31- \\
1.12)\end{array}$ & 0.11 \\
\hline \multicolumn{9}{|l|}{ Age (years) } \\
\hline$<65$ & $\begin{array}{l}22229 \\
(85.4 \%)\end{array}$ & 39227.5 & 13 & 33.1 & $\begin{array}{l}(17.6- \\
56.7)\end{array}$ & & & \\
\hline$\geq 65$ & $\begin{array}{l}3793 \\
(14.6 \%)\end{array}$ & 5346.9 & 30 & 561.1 & $\begin{array}{l}(378.6- \\
801.0)\end{array}$ & 17.41 & $\begin{array}{l}(8.70- \\
34.83)\end{array}$ & $<0.01$ \\
\hline \multicolumn{9}{|l|}{ Ethnicity } \\
\hline Han & $\begin{array}{l}24107 \\
(92.6 \%)\end{array}$ & 41304.6 & 36 & 87.2 & $\begin{array}{l}(61.0- \\
120.7)\end{array}$ & ref & & \\
\hline Other minority & $\begin{array}{l}1915 \\
(7.4 \%)\end{array}$ & 3269.8 & 7 & 214.1 & $\begin{array}{l}(86.1- \\
441.1)\end{array}$ & 2.27 & $\begin{array}{l}(0.94- \\
5.50)\end{array}$ & 0.07 \\
\hline \multicolumn{9}{|l|}{ Education level } \\
\hline Illiterate or semi-illiterate & $\begin{array}{l}2048 \\
(7.9 \%)\end{array}$ & 3134.0 & 14 & 446.7 & $\begin{array}{l}(244.2- \\
749.5)\end{array}$ & ref & & \\
\hline Primary school & $\begin{array}{l}4671 \\
(18.0 \%)\end{array}$ & 7686.4 & 13 & 169.1 & $\begin{array}{l}(90.1- \\
289.2)\end{array}$ & 0.38 & $\begin{array}{l}(0.17- \\
0.85)\end{array}$ & 0.02 \\
\hline Secondary school & $\begin{array}{l}15126 \\
(58.1 \%)\end{array}$ & 26416.3 & 13 & 49.2 & $\begin{array}{l}(26.2- \\
84.2)\end{array}$ & 0.11 & $\begin{array}{l}(0.05- \\
0.25)\end{array}$ & $<0.01$ \\
\hline College and above & $\begin{array}{l}4123 \\
(15.8 \%)\end{array}$ & 7248.5 & 3 & 41.4 & $\begin{array}{l}(8.5- \\
121.0)\end{array}$ & 0.08 & $\begin{array}{l}(0.02- \\
0.32)\end{array}$ & $<0.01$ \\
\hline Unknown & $54(0.2 \%)$ & 89.2 & 0 & 0.0 & $\begin{array}{l}(0.0- \\
4133.7)\end{array}$ & - & & \\
\hline \multicolumn{9}{|l|}{ Marital status } \\
\hline Married & $\begin{array}{l}19262 \\
(74.0 \%)\end{array}$ & 33163.6 & 26 & 78.4 & $\begin{array}{l}(51.2- \\
114.9)\end{array}$ & ref & & \\
\hline Single & $\begin{array}{l}4179 \\
(16.1 \%)\end{array}$ & 7280.2 & 3 & 41.2 & $\begin{array}{l}(8.5- \\
120.4)\end{array}$ & 0.42 & $\begin{array}{l}(0.10- \\
1.67)\end{array}$ & 0.22 \\
\hline Widowed/divorced/separated/other & $\begin{array}{l}2581 \\
(9.9 \%)\end{array}$ & 4130.7 & 14 & 338.9 & $\begin{array}{l}(185.3- \\
568.7)\end{array}$ & 4.23 & $\begin{array}{l}(2.12- \\
8.45)\end{array}$ & $<0.01$ \\
\hline \multicolumn{9}{|l|}{$\begin{array}{l}\text { Family annual income per capita } \\
\text { (Median }=7200 \mathrm{RMB} \text { ) }\end{array}$} \\
\hline Below the median & $\begin{array}{l}12975 \\
(49.9 \%)\end{array}$ & 22547.9 & 20 & 88.7 & $\begin{array}{l}(54.2- \\
137.0)\end{array}$ & ref & & \\
\hline Above the median & $\begin{array}{l}13047 \\
(50.1 \%)\end{array}$ & 22026.5 & 23 & 104.4 & $\begin{array}{l}(66.2- \\
156.7)\end{array}$ & 1.18 & $\begin{array}{l}(0.63- \\
2.23)\end{array}$ & 0.60 \\
\hline \multicolumn{9}{|l|}{ Previously treated TB } \\
\hline No & $\begin{array}{l}25884 \\
(99.5 \%)\end{array}$ & 44368.3 & 38 & 85.6 & $\begin{array}{l}(60.6- \\
117.6)\end{array}$ & & & \\
\hline Yes & $138(0.5 \%)$ & 206.2 & 5 & 2425.3 & $\begin{array}{l}\text { (787.5- } \\
5659.8)\end{array}$ & 25.01 & $\begin{array}{l}(8.84- \\
70.78)\end{array}$ & $<0.01$ \\
\hline
\end{tabular}

Abbreviations: Cl, Confidence interval; HIV, Human immunodeficiency virus; AIDS, Acquired immune deficiency syndrome; BCG, Bacillus CalmetteGuerin vaccine 


\begin{tabular}{|c|c|c|c|c|c|c|c|c|}
\hline \multirow[t]{2}{*}{ Characteristics } & \multirow{2}{*}{$\begin{array}{l}\begin{array}{l}\text { Number of } \\
\text { participants } \\
\text { in cohort }\end{array} \\
\mathrm{n}(\%)\end{array}$} & \multirow[t]{2}{*}{$\begin{array}{l}\text { Person- } \\
\text { years }\end{array}$} & \multirow{2}{*}{$\begin{array}{l}\begin{array}{l}\text { Number } \\
\text { of } \\
\text { cases }\end{array} \\
\mathrm{n} \\
\end{array}$} & \multicolumn{2}{|c|}{$\begin{array}{l}\text { Incident rate } \\
\text { (per } 100 \text { 000) }\end{array}$} & \multicolumn{2}{|c|}{$\begin{array}{l}\text { Incident rate } \\
\text { ratio }\end{array}$} & \multirow[t]{2}{*}{$p$-value } \\
\hline & & & & $(95 \% \mathrm{Cl}$ & & $(95 \%$ & & \\
\hline \multicolumn{9}{|l|}{ Close contacts } \\
\hline No & $\begin{array}{l}25989 \\
(99.9 \%)\end{array}$ & 44518.7 & 43 & 96.6 & $\begin{array}{l}(70.1- \\
130.5)\end{array}$ & & & \\
\hline Yes & $33(0.1 \%)$ & 55.7 & 0 & 0.0 & $\begin{array}{l}(0.0- \\
6624.0)\end{array}$ & - & & \\
\hline \multicolumn{9}{|l|}{ Known HIV/AIDS } \\
\hline No & $\begin{array}{l}26001 \\
(99.9 \%)\end{array}$ & 44547.2 & 43 & 96.5 & $\begin{array}{l}(69.9- \\
130.0)\end{array}$ & & & \\
\hline Yes & $21(0.1 \%)$ & 27.3 & 0 & 0.0 & $\begin{array}{l}(0.0- \\
13532.2)\end{array}$ & - & & \\
\hline \multicolumn{9}{|l|}{ Known diabetes } \\
\hline No & $\begin{array}{l}25383 \\
(97.5 \%)\end{array}$ & 43657.4 & 41 & 93.9 & $\begin{array}{l}(67.4- \\
127.4)\end{array}$ & ref & & \\
\hline Yes & $639(2.5 \%)$ & 917.1 & 2 & 218.1 & $\begin{array}{l}(26.4- \\
787.8)\end{array}$ & 1.56 & $\begin{array}{l}(0.28- \\
8.76)\end{array}$ & 0.62 \\
\hline \multicolumn{9}{|c|}{ Chronic bronchitis } \\
\hline No & $\begin{array}{l}24837 \\
(95.4 \%)\end{array}$ & 42653.9 & 41 & 96.1 & $\begin{array}{l}(69.0- \\
130.4)\end{array}$ & ref & & \\
\hline Yes & 919 (3.5\%) & 1455.1 & 2 & 137.4 & $\begin{array}{l}(16.6- \\
496.5)\end{array}$ & 0.96 & $\begin{array}{l}(0.17- \\
5.39)\end{array}$ & 0.96 \\
\hline Unknown & $266(1.0 \%)$ & 465.4 & 0 & 0.0 & $\begin{array}{l}(0.0- \\
792.6)\end{array}$ & - & & \\
\hline \multicolumn{9}{|l|}{ Pneumoconiosis } \\
\hline No & $\begin{array}{l}25955 \\
(99.7 \%)\end{array}$ & 44475.3 & 42 & 94.4 & $\begin{array}{l}(68.1- \\
127.6)\end{array}$ & ref & & \\
\hline Yes & $67(0.3 \%)$ & 99.1 & 1 & 1009.2 & $\begin{array}{l}(25.6- \\
5622.8)\end{array}$ & 4.07 & $\begin{array}{l}(0.27- \\
61.44)\end{array}$ & 0.31 \\
\hline \multicolumn{9}{|l|}{ Smoking status } \\
\hline Never & $\begin{array}{l}19308 \\
(74.2 \%)\end{array}$ & 32995.7 & 28 & 84.9 & $\begin{array}{l}(56.4- \\
122.6)\end{array}$ & ref & & \\
\hline Former & $822(3.2 \%)$ & 1318.3 & 1 & 75.9 & $\begin{array}{l}(1.9- \\
422.7)\end{array}$ & 0.34 & $\begin{array}{l}(0.02- \\
5.23)\end{array}$ & 0.44 \\
\hline Current & $\begin{array}{l}5892 \\
(22.6 \%)\end{array}$ & 10260.4 & 14 & 136.4 & $\begin{array}{l}(74.6- \\
228.9)\end{array}$ & 1.57 & $\begin{array}{l}(0.79- \\
3.11)\end{array}$ & 0.19 \\
\hline \multicolumn{9}{|l|}{ Alcohol use } \\
\hline Never & $\begin{array}{l}20532 \\
(78.9 \%)\end{array}$ & 35082.0 & 27 & 77.0 & $\begin{array}{l}(50.7- \\
112.0)\end{array}$ & ref & & \\
\hline Former & $\begin{array}{l}1066 \\
(4.1 \%)\end{array}$ & 1772.5 & 5 & 282.1 & $\begin{array}{l}(91.6- \\
658.3)\end{array}$ & 3.26 & $\begin{array}{l}(1.13- \\
9.43)\end{array}$ & 0.03 \\
\hline Current & $\begin{array}{l}4424 \\
(17.0 \%)\end{array}$ & 7719.9 & 11 & 142.5 & $\begin{array}{l}(71.1- \\
255.0)\end{array}$ & 1.79 & $\begin{array}{l}(0.84- \\
3.79)\end{array}$ & 0.13 \\
\hline \multicolumn{9}{|l|}{ BCG scar } \\
\hline Yes & $\begin{array}{l}15301 \\
(58.8 \%)\end{array}$ & 26975.3 & 11 & 40.8 & $\begin{array}{l}(20.4- \\
73.0)\end{array}$ & ref & & \\
\hline No & $\begin{array}{l}9127 \\
(35.1 \%)\end{array}$ & 14772.3 & 29 & 196.3 & $\begin{array}{l}(131.5- \\
281.9)\end{array}$ & 5.00 & $\begin{array}{l}(2.38- \\
10.51)\end{array}$ & $<0.01$ \\
\hline Unknown & $\begin{array}{l}1594 \\
(6.1 \%)\end{array}$ & 2826.8 & 3 & 106.1 & $\begin{array}{l}(21.9- \\
310.1)\end{array}$ & 2.14 & $\begin{array}{l}(0.49- \\
9.31)\end{array}$ & 0.31 \\
\hline
\end{tabular}


Table 3

Body mass index and risks of tuberculosis in the cohort.

\begin{tabular}{|c|c|c|c|c|c|c|}
\hline \multicolumn{7}{|c|}{ BMI category, kg/m² } \\
\hline & \multicolumn{2}{|c|}{ Underweight } & \multicolumn{2}{|c|}{ Normal } & \multicolumn{2}{|c|}{ Overweight and obese } \\
\hline & \multicolumn{2}{|l|}{$<18.5$} & \multicolumn{2}{|c|}{18.5 to 24.0} & \multicolumn{2}{|l|}{$\geq 24.0$} \\
\hline Mean $(95 \% C l), \mathrm{kg} / \mathrm{m}^{2}$ & 17.5 & $(17.4-17.5)$ & 21.4 & $(21.3-21.4)$ & 26.3 & $(26.2-26.3)$ \\
\hline Person-years & 3464.8 & & 27382. & & 13727.7 & \\
\hline Number of cases & 6 & & 31 & & 6 & \\
\hline Incidence rate (per 100 000, 95\% Cl) & 173.2 & $(63.6-376.9)$ & 113.2 & $(76.9-160.7)$ & 43.7 & $(16.0-95.1)$ \\
\hline Incident rate ratio $(95 \% \mathrm{Cl})$ & 1.39 & $(0.53-3.65)$ & ref & & $0.35^{\dagger}$ & $(0.13-0.92)$ \\
\hline $\operatorname{cHR}(95 \% \mathrm{Cl})$ & 1.55 & $(0.65-3.71)$ & ref & & $0.36^{\dagger}$ & $(0.15-0.87)$ \\
\hline Age-adjusted $H R(95 \% C l)^{a}$ & 1.26 & $(0.52-3.03)$ & ref & & $0.34^{\dagger}$ & $(0.14-0.82)$ \\
\hline Multivariable adjusted $H R(95 \% C l)^{\mathrm{b}}$ & 1.26 & $(0.52-3.06)$ & ref & & $0.34^{\dagger}$ & $(0.14-0.82)$ \\
\hline Corrected with DM $H R(95 \% C l)^{\mathrm{c}}$ & 1.25 & $(0.52-3.04)$ & ref & & $0.34^{\dagger}$ & $(0.14-0.83)$ \\
\hline \multicolumn{7}{|c|}{ Abbreviations: BMI, Body mass index; Cl, Confidence interval; HR, Hazard ratio, cHR, crude Hazard ratio; DM, Diabetes mellitus } \\
\hline \multicolumn{7}{|c|}{ a Age-adjusted $H R$ : category with aged 65 years. } \\
\hline \multicolumn{7}{|c|}{$\begin{array}{l}\text { b Multivariable adjusted } H R \text { : after variable selection, adjusted with covariates: age, sex, ethnicity, marital status, previously treated TB, smoking status, } \\
\text { alcohol use, all variables were adjusted as categorical variable. }\end{array}$} \\
\hline \multicolumn{7}{|c|}{$\begin{array}{l}\text { ' Corrected with DM HR: adjusted with covariates: age, sex, ethnicity, marital status, previously treated TB, smoking status, alcohol use and known } \\
\text { diabetes, all variables were adjusted as categorical variable. }\end{array}$} \\
\hline${ }^{\dagger} p<0.01$ & & & & & & \\
\hline
\end{tabular}


Table 4

Subgroup analysis for the association between body mass index and risks of tuberculosis in the cohort.

\begin{tabular}{|c|c|c|c|c|c|c|c|c|c|}
\hline Covariates & BMI groups & $\begin{array}{l}\text { Person- } \\
\text { years }\end{array}$ & $\begin{array}{l}\text { Number of } \\
\text { cases }\end{array}$ & Incider & e rate & $a H R$ & & $p$ & Interaction \\
\hline & & & n & (95\% C & & $(95 \%$ & & & pvalue \\
\hline $\operatorname{Sex}^{\mathrm{a}}$ & & & & & & & & & \\
\hline Male & Underweight & 1408.3 & 5 & 355.0 & $\begin{array}{l}(115.3- \\
828.6)\end{array}$ & 1.69 & $\begin{array}{l}(0.59- \\
4.82)\end{array}$ & 0.33 & ref \\
\hline & Normal & 12370.7 & 17 & 137.4 & $\begin{array}{l}(80.1- \\
220.0)\end{array}$ & ref & & & \\
\hline & Overweight & 7452.7 & 4 & 53.7 & $(14.6-137.4)$ & 0.44 & $\begin{array}{l}(0.15- \\
1.31)\end{array}$ & 0.14 & ref \\
\hline Female & Underweight & 2056.5 & 1 & 48.6 & $(1.2-270.9)$ & 0.38 & $\begin{array}{l}(0.05- \\
2.93)\end{array}$ & 0.35 & 0.25 \\
\hline & Normal & 15011.2 & 14 & 93.3 & $(51.0-156.5)$ & ref & & & \\
\hline & Overweight & 6274.9 & 2 & 31.9 & $(3.9-115.1)$ & 0.22 & $\begin{array}{l}(0.05- \\
0.97)\end{array}$ & 0.05 & 0.52 \\
\hline $\begin{array}{l}\text { Age } \\
\text { (years) }^{b}\end{array}$ & & & & & & & & & \\
\hline$<65$ & Underweight & 2981.1 & 2 & 67.1 & $(8.1-242.3)$ & 1.79 & $\begin{array}{l}(0.37- \\
8.65)\end{array}$ & 0.47 & ref \\
\hline & Normal & 24229.7 & 9 & 37.1 & $(17.0-70.5)$ & ref & & & \\
\hline & Overweight & 12016.6 & 2 & 16.6 & $(2.0-60.1)$ & 0.42 & $\begin{array}{l}(0.09- \\
2.00)\end{array}$ & 0.28 & ref \\
\hline$\geq 65$ & Underweight & 483.7 & 4 & 827.0 & $\begin{array}{l}(225.3- \\
2117.6)\end{array}$ & 1.20 & $\begin{array}{l}(0.41- \\
3.54)\end{array}$ & 0.74 & 0.54 \\
\hline & Normal & 3152.3 & 22 & 697.9 & $\begin{array}{l}(437.4- \\
1056.7)\end{array}$ & ref & & & \\
\hline & Overweight & 1711.0 & 4 & 233.8 & $(63.7-598.6)$ & 0.36 & $\begin{array}{l}(0.12- \\
1.00)\end{array}$ & 0.05 & 0.87 \\
\hline Abbreviatior & BMI, Body ma & ex; $\mathrm{Cl}, \mathrm{Co}$ & nterval; aHR & Hazar & ratio & & & & \\
\hline $\begin{array}{l}\text { a Adjusted n } \\
\text { variable. }\end{array}$ & covariates: a & nicity, ma & Is, previous & B, sm & ng status, alcc & e, all v & iables v & djustec & ategorical \\
\hline $\begin{array}{l}\text { b Adjusted n } \\
\text { variable. }\end{array}$ & covariates: s & nicity, ma & us, previousl) & $\mathrm{B}, \mathrm{sm}$ & ing status, alcc & e, all & iables & djuste & tegorical \\
\hline
\end{tabular}

\section{Results}

\section{Baseline characteristics and follow-up of the cohort}

This study included 26022 participants and followed up to outcomes. The underweight, normal weight and overweight or obese participants occupied the proportion of $7.9 \%, 61.5 \%$, and $30.6 \%$ at baseline, respectively. The median age was 44 years and the male to female ratio was 1 to 1.1 (Table 1 ).

The followed-up time of the cohort was 2.25 years ( 27 months) in three rounds of screening. Overall, the number of 43 cases developed tuberculosis in 44 574.4 observed person-years, the tuberculosis incidence rate was 96.5 per 100000 person-years ( $95 \% \mathrm{Cl} 69.8-129.9)$. Median times to incident tuberculosis was 1.64 years (20 months). Tuberculosis incidence was significantly higher in those aged over 65 years, lower education level, widowed/divorced/separated/other, previously treated tuberculosis, former drinker, and persons without BCG scar (Table 2).

\section{Dose-response relationship of BMI and tuberculosis incident}

The Kaplan-Meier univariate analysis showed that the cumulative hazard of developing tuberculosis was different by BMI levels (the overall Log-Rank test $p=0.021$ ). Lower BMI had a shorter time of developing tuberculosis (Fig. 2). 
For the cohort participants, the incidence rate of tuberculosis was 173.2 (95\% Cl 63.6-376.9), 113.2 (95\% Cl 76.9-160.7), and 43.7 (95\% Cl 16.0-95.1) per 100000 person-years in underweight, normal weight and overweight or obese respectively (Table 3).

Inverse logarithmic-linear dose-response relationship between BMI and tuberculosis incidence was fitted. The logarithmic transferred TB incidence and linear BMI change were regressed $\left(R^{2}=0.98\right.$, adjusted $\left.R^{2}=0.95\right)$, the intercept and slope were 3.46 and -0.068 respectively (Fig. 3).

\section{Dose-response relationship of BMI and tuberculosis risk}

After adjusting the covariates, the multivariable Cox proportional regression presented that overweight and obese was associated with a lower hazard of incident tuberculosis compared with normal weight (aHR, $0.34 ; 95 \% \mathrm{Cl} 0.14-0.82, p<0.01)$, underweight related to a higher hazard but statistical nonsignificant (aHR, 1.26; 95\% Cl 0.52-3.06, $p=0.60)$. The BMI linear trend was significant and an estimated $48 \%$ reduction for tuberculosis hazard along with $\mathrm{BMI}$ increased (aHR, $0.52 ; 95 \% \mathrm{Cl} 0.31-0.85, p<0.01)$. After correction with DM status, the association between $\mathrm{BMI}$ and tuberculosis risk remained unchanged (Table 3).

Overall and in subgroups, restricted cubic spline regressions were fitted to present dose-response relationship between continuous BMI and linear cumulative hazards of tuberculosis (Figs. 4 and 5). Approximated negative linear or cubic polynomial relationship between the pooled or stratified risks of tuberculosis and BMI, except the subgroups of those single or former drinkers, showed a U-shaped curve.

\section{Subgroups analysis according to gender and age}

Across different subgroups of sex, tuberculosis incidence inversely related to BMI in male induvial. The risk of tuberculosis showed a $78 \%$ reduction in overweight or obese (aHR, $0.22 ; 95 \% \mathrm{Cl} 0.05-0.97, p=0.05$ ) compared with normal weight among females (Table 4). While stratified by age under or above 65 , tuberculosis incidence inversely related to BMI in both populations. The hazard of tuberculosis presented a $64 \%$ reduction in overweight or obese (aHR, $0.36 ; 95 \% \mathrm{Cl} 0.12-1.00, p=0.05)$ compared with normal weight among the elderly. There was no evidence of interaction effect for BMI by sex or age.

\section{Discussion}

In this prospective population-based cohort study, we estimated the inverse log-linear dose-response association of BMI and the tuberculosis incidence in contemporary Chinese adults. The cohort study clarified the higher BMI was a strong predictor for tuberculosis development, a two-third reduction of tuberculosis risk for overweight and obese compared with normal weight population, protective effect yet presented in sub-population of female and elderly. Additionally, consistent inverse relationships were precisely illustrated between the successive BMI and the risk of tuberculosis overall and stratified.

The log-linear inverse association between the BMI and tuberculosis incidence was consistent with the result of the previous systemic review [3]. Though five out of six cohort studies included in this systemic review were performed in the last century, and all 6 studies were carried out in high-income counties. Likewise, large population-based cohorts reported by Cegielski et al in the US [8] and Pealing et al in the UK [9] stated the inverse association in low disease burden and high-income setting. Our study was implemented in the high burden site of a middle-income country, this inverse association remained unchanged to these reports far distance from recent in lower TB burden settings.

Most recently, studies presented the BMI related to the tuberculosis development. Population-based observational studies revealed that lower BMI was related to tuberculosis. Researches of the cross-sectional prevalence investigation and followed up cohort among elderly in China, Zhang et al and Cheng et al $[10,11]$ reported that a significantly higher risk or hazard of tuberculosis in the underweight group (adjusted odds ratio [aOR], 1.55; 95\% $\mathrm{Cl}, 1.09-2.22$ ) and (aHR, 2.33; 95\% Cl 1.32-4.12) respectively, compared with normal-weight among those age over 65 years in China. However, our study reported a nonsignificant association for underweight and tuberculosis in general adult and elderly sub-population. This discrepancy might be trigger by the difference proportion and distribution of baseline demographic and socioeconomic factors, meanwhile, this previous elderly cohort study reported a large proportion of loss-to-follow might overestimate the risk.

Besides, many shreds of evidence presented overweight or obesity was associated with a lower risk of tuberculosis. Yen et al [12] reported in a Taiwan cohort that by comparing with normal weight group, the overweight (aOR, 0.67; 95\% Cl, 0.49-0.91) and obesity $(\mathrm{aOR}, 0.43 ; 95 \% \mathrm{Cl}, 028-0.67)$ was a protective factor to tuberculosis incident. Kim et al [13] reported a consistent inverse association between BMI and tuberculosis, this followed up research also presented that overweight (aHR, $0.45 ; 95 \% \mathrm{Cl}, 0.40-0.50)$ and obesity (aHR, $0.40 ; 95 \% \mathrm{Cl}, 0.30-0.54)$ as a protective predictor of tuberculosis. Although there was a slight difference in hazard or risk ratio for tuberculosis development among studies, regarding the cutoff points for BMI categorization and the definition for overweight or obesity was different across study sites and countries, all these researches revealed the identical direction of association with our study. These unanimous results from systemic review and multi-population observational studies, with addition to this cohort among Chinese adulthood, showed the strong and constant evidence of the inverse association between BMI and tuberculosis incidence.

The highlight of the present study was that we quantified the dose-response relationship between BMI and the disease incident, as well as the risk of tuberculosis progression. Our research established the association between successive BMI and TB incident, which was more accurate and solid compared with previous discrete BMI levels. Under the high burden of 96.5 per 100000 person-years incident setting, the logarithmic TB incidence decreased response to the linear increment of BMI. The results suggested that the tiny improvement of population nutrition status would have a great 
public health impact that significantly decreased the TB incident, especially among nutritionally vulnerable populations such as BMI under $18.5 \mathrm{~kg} / \mathrm{m}^{2}$. For instance, the BMI increased from 18 to $19 \mathrm{~kg} / \mathrm{m}^{2}$, the TB incidence declined 24.8 per 100000 person-years in population. This suggests that the nutritionimprovement food distribution program for the targeted population might be a beneficial strategy for these high burden regions. This also implied that the increment of main exposure BMI during decades might play a crucial role in the overall $28 \%$ decline of tuberculosis prevalence between 1990 and 2010 in China [14].

There was another contribution to this study. We had got a better understanding of the logarithmic predicted individual tuberculosis risk declined accompanied by BMI linear increment, despite subgroups such as single person or former drinker presented U-shaped curve. The BMI value of $22.5 \mathrm{~kg} / \mathrm{m}^{2}$ was on the log-linear $\mathrm{x}$-axis (with slope and intercept of zero) that corresponded to an exponential tuberculosis hazard of 1 . This implied the cutoff point of $\mathrm{BMI}$ on tuberculosis hazard was $22.5 \mathrm{~kg} / \mathrm{m}^{2}$, the value was the threshold of hazardous-protective effect for tuberculosis progression among the general community population. The potential benefit of this novel insight was that while implementing the tuberculosis active case finding (ACF) screening, we speculated the screening strategy could possibly yield if sub-population of BMI under $22.5 \mathrm{~kg} / \mathrm{m}^{2}$ was set as one of the targeted populations [6, 15]. Notwithstanding, the discussion about the universal BMI category was inappropriate. Disease morbidity-, disease mortality-, or population-specific BMI categorization was reasonable [16], many studies confirmed a lower BMI cutoff point for Chinese adults to define fat, overweight or obese while considering comorbidities, progression or sub-population [17-20]. The generalization of this finding should be cautious because the cohort sample was unrepresentative to other regions, more evidence was needed to confirm the cutoff point of BMI and the yield of screening.

DM was a well-known risk factor for tuberculosis, BMI also had a strong relation with DM. Although in the present study the association between known DM and TB was no-significant in the DM corrected model, the reduction of TB risk associated with BMI remains unchanged after including DM status. A systemic review of 3 cohorts examined DM associated with an increased risk of TB (relative risk, 3.11; 95\% Cl 2.27-4.26) [21]. Kuo et al [22] reported type 2 diabetes (T2DM) significantly increase $31 \%$ tuberculosis risk (aHR, 1.31; 95\% $\mathrm{Cl} 1.23-1.39$ ) after adjusting sex, age, and comorbidities. Lee et al [23] then revealed DM patients with poor glycemic control had a higher hazard of TB (aHR, 2.21; 95\% Cl 1.63-2.99) after adjusting covariates and BMI. Lin et al [24] stated causal mediation between factors of BMI, DM, and TB. Obesity decreased tuberculosis hazard in 2 cohorts followed up over 7 years, obesity had a slight hazard effect on tuberculosis mediated by DM but had a strong protective effect not mediated through DM. A feasible explanation for this argument and inconsistent was that the prevalence of DM in the present cohort was $2.5 \%$, which was substantially lower than $9.7 \%$ of total diabetes prevalence among China adults [25]. The evidence was insufficient of DM as a risk factor or its interaction effect with BMI for tuberculosis incidence in lower prevalence during the short-term observation.

The biological mechanism of BMI effect on tuberculosis incident was complicated. The population nutrition status was characterized mainly by the indicator of BMI. Nutrition, immune function, and infection were complex related and in dynamic interact patterns. The malnutrition linked to the cellmediated immunity (CMI) system that was profoundly affected the host defense against Mycobacterium tuberculosis, result in the tuberculosis infection or immunity. Malnourished mice caused chronic Protein-energy malnutrition (PEM) suffered a higher bacterial burden and the efficacy of BCG vaccination was reduced [26]. A review stated that PEM impairs $\mathrm{CMI}$ and worsens the infections, conversely, the infection can lead rapidly to weight loss, malnutrition, and immunologic dysfunction, moreover, PEM is partly due to food deprivation [27]. Thus, the direction and interact or causal relationship between malnutrition and tuberculosis still scarcely understood, many unknown variables affect the relationship. Another possible interpretation was that the difference in genetic susceptibility of obesity among hosts leads to the association. Obesity-associated gene FTO (fat mass and obesity-associated, Gene ID: 79068) and its genetic polymorphisms rs9939609, rs8050136 within the FTO associated with obesity. The significant association between the genetic polymorphism rs9939609 and tuberculosis risk was found, that compared with the common genotype TT, individuals carrying AA had a tuberculosis risk (aOR, 3.77; 95\% Cl: 2.26-6.28) [28].

The strengthening of our research is that this population-based prospective study included a large number of community participants in the southwestern mountainous region of China. The cohort estimated steady tuberculosis incidence under relatively high disease burden setting in Chinese adulthood, which could supply solid evidence for tuberculosis control policymaker in the area. Moreover, this well-designed cohort study quantified log-linear inverse doseresponse relationship between $\mathrm{BMI}$ and tuberculosis incidence, yet estimated the dose-response association between BMI and individual risk progress to tuberculosis. Additionally, the overall and stratification analysis was performed after adjusting the confounders. The protective effect of the higher BMI presented in the general population and subgroup of females and the elderly might contribute to TB control policy.

However, there were a few limitations to the study. First, this study followed up over two years and conducted three rounds of screening due to the limited funding and resource. Accordingly, we could not observe all the tuberculosis incidents in a long period, the short-term followed up period plus the low prevalence of comorbidities at baseline (such as known DM, known HIV/AIDS, Chronic bronchitis, Pneumoconiosis) might lead to these factors insignificant associated with tuberculosis development. A systemic review showed that in the first 2 years after latent tuberculosis infection (LTBI) status, $65 \%$ of the LTBI cases progressed to active tuberculosis in a total follow up time of 20 years [29]. This cohort could capture most of the LTBI development to tuberculosis, but there were still 35\% infected individual could not reach end-point of disease progression due to the short-term follow up. Second, the study did not get LTBI status in the baseline population, how the BMI effect on LTBI progression to active TB was still unsolved. Though there was evidence that the lower BMI did not increase the LTBI risk [30], overweight or obesity was a risk factor associated with LTBI status [31]. Further study should be conducted to clarify this interesting contradiction of BMI playing a different role in LTBI status and TB development. Additionally, several covariates were self-reported by participants such as smoking and drinking habit, the exposure level without accurate measurement would be imprecise to estimate the association.

\section{Conclusion}


Collectively, the study revealed the inverse log-linear dose-response relationship between $\mathrm{BMI}$ and incident tuberculosis, likewise the association between $\mathrm{BMI}$ and risk of tuberculosis progression in contemporary Chinese adulthood. Overweight and obesity was the protective factor of TB development in the general population and subgroups of female or elderly.

\section{Abbreviations}

BMl:body mass index; TB:tuberculosis; PTB:pulmonary tuberculosis; aHR:adjusted hazard ratio; Cl:confidence interval; SEVs:summary exposure values; CHWs:community health workers; BCG:Bacillus Calmette-Guerin; TBIMS:Tuberculosis Information Management System; HIV/AIDS:human immunodeficiency virus or acquired immune deficiency syndrome; CDC:Center for Disease Control and Prevention; DM:diabetes mellitus; BPHS:National Project of Basic Public Health Service; CXR:chest X-ray; IR:incident rate; IRR:incident rate ratio; RCS:restricted cubic spline; IRB:Institutional Review Board; aOR:adjusted odds ratio; ACF:active case finding; T2DM:type 2 diabetes; CMI:cell-mediated immunity; PEM:protein-energy malnutrition; LTBI:latent tuberculosis infection.

\section{Declarations}

\section{Ethical Approval}

This study was reviewed and approved by the Institutional Review Board (IRB) of the Chinese Center for Disease Control and Prevention (No. 201820). All participants gave written consent to enroll and participate in this study. The study was not included personal identification and all data in analyses was anonymized.

\section{Competing Interests}

The authors declare that they have no competing interests.

\section{Funding}

The primary study "Study on TB epidemic and intervention mode" was funded by The National Twelfth Five-year Mega-Scientific Projects of Infectious Diseases in China (Grant No. 2013ZX10003-004 - 001). The funders had no role in study design, data collection and analysis, writing of the manuscript and decision to publish

\section{Authors' Contributions}

JC, SZ and LX conceived and designed the study. JC, SZ, JH, KL, YQ, RY, LL and YY collected the data. JC performed the statistical analysis. JC and LX prepared the manuscript. All authors read and approved the final manuscript.

\section{Acknowledgments}

We thank the tireless contributions of staff in Dongchuan CDC, Kunming CDC, and other related health care workers in undertaking the cohort in Dongchuan County. We thank TB department colleagues for their assistance in data collection.

\section{Consent for publication}

Not applicable.

\section{Availability of Data and Material}

We do not wish to share our data. Data is available upon reasonable request to the corresponding author.

\section{References}

1. Kyu HH, Maddison ER, Henry NJ, Mumford JE, Barber R, Shields C, et al. The global burden of tuberculosis: results from the Global Burden of Disease Study 2015. Lancet Infect Dis. 2018;18:261-84.

2. World Health Organization. Global tuberculosis report 2019. Geneva; 2019.

3. Lonnroth K, Williams BG, Cegielski P, Dye C. A consistent log-linear relationship between tuberculosis incidence and body mass index. Int J Epidemiol. 2010;39:149-55.

Page 12/18 
4. NCD Risk Factor Collaboration (NCD-RisC). Trends in adult body-mass index in 200 countries from 1975 to 2014 : a pooled analysis of 1698 population-based measurement studies with 19.2 million participants. Lancet. 2016;387:1377-96.

5. Zhou M, Wang H, Zeng X, Yin P, Zhu J, Chen W, et al. Mortality, morbidity, and risk factors in China and its provinces, 1990-2017: a systematic analysis for the Global Burden of Disease Study 2017. Lancet. 2019;394:1145-58.

6. Chen J-O, Qiu Y-B, Rueda ZV, Hou J-L, Lu K-Y, Chen L-P, et al. Role of community-based active case finding in screening tuberculosis in Yunnan province of China. Infect Dis Poverty. 2019;8:92.

7. Ministry of Health of the People's Republic of China. Criteria of weight for adults(WS/T428-2013). Ministry of Health of the People's Republic of China. 2013.

8. Cegielski JP, Arab L, Cornoni-Huntley J. Nutritional Risk Factors for Tuberculosis Among Adults in the United States, 1971-1992. Am J Epidemiol. 2012;176:409-22.

9. Pealing L, Wing K, Mathur R, Prieto-Merino D, Smeeth L, Moore DAJ. Risk of tuberculosis in patients with diabetes: population based cohort study using the UK Clinical Practice Research Datalink. BMC Med. 2015;13:135.

10. Zhang C-Y, Zhao F, Xia Y-Y, Yu Y-L, Shen X, Lu W, et al. Prevalence and risk factors of active pulmonary tuberculosis among elderly people in China: a population based cross-sectional study. Infect Dis Poverty. 2019;8:7.

11. Cheng J, Sun Y-N, Zhang C-Y, Yu Y-L, Tang L-H, Peng H, et al. Incidence and risk factors of tuberculosis among the elderly population in China: a prospective cohort study. Infect Dis Poverty. 2020;9:13.

12. Yen Y-F, Hu H-Y, Lee Y-L, Ku P-W, Lin I-F, Chu D, et al. Obesity/overweight reduces the risk of active tuberculosis: a nationwide population-based cohort study in Taiwan. Int J Obes. 2017;41:971-5.

13. Kim SJ, Ye S, Ha E, Chun EM. Association of body mass index with incident tuberculosis in Korea. PLoS One. 2018;13:e0195104.

14. Wang L, Zhang H, Ruan Y, Chin DP, Xia Y, Cheng S, et al. Tuberculosis prevalence in China, 1990-2010; a longitudinal analysis of national survey data. Lancet. 2014;383:2057-64.

15. Zhang C, Ruan Y, Cheng J, Zhao F, Xia Y, Zhang H, et al. Comparing yield and relative costs of WHO TB screening algorithms in selected risk groups among people aged 65 years and over in China, 2013. PLoS One. 2017;12:e0176581.

16. Deurenberg P. Universal cut-off BMI points for obesity are not appropriate. Br J Nutr. 2001;85:135-6.

17. He W, Li Q, Yang M, Jiao J, Ma X, Zhou Y, et al. Lower BMI cutoffs to define overweight and obesity in China. Obesity. 2015;23:684-91.

18. Zeng Q, He Y, Dong S, Zhao X, Chen Z, Song Z, et al. Optimal cut-off values of BMI, waist circumference and waist:height ratio for defining obesity in Chinese adults. Br J Nutr. 2014;112:1735-44.

19. Pei X, Liu L, Cai Y, Peng Y, Ma C, Jin Y, et al. Body mass index cut-off points for predicting chronic non-communicable disease should differ by gender and age group. Public Health. 2019;175:54-9.

20. Wildman RP, Gu D, Reynolds K, Duan X, He J. Appropriate body mass index and waist circumference cutoffs for categorization of overweight and central adiposity among Chinese adults. Am J Clin Nutr. 2004;80:1129-36.

21. Jeon CY, Murray MB. Diabetes Mellitus Increases the Risk of Active Tuberculosis: A Systematic Review of 13 Observational Studies. PLoS Med. 2008;5:e152.

22. Kuo M-C, Lin S-H, Lin C-H, Mao I-C, Chang S-J, Hsieh M-C. Type 2 Diabetes: An Independent Risk Factor for Tuberculosis: A Nationwide PopulationBased Study. PLoS One. 2013;8:e78924.

23. Lee P-H, Fu H, Lai T-C, Chiang C-Y, Chan C-C, Lin H-H. Glycemic Control and the Risk of Tuberculosis: A Cohort Study. PLOS Med. $2016 ; 13: e 1002072$.

24. Lin H-H, Wu C-Y, Wang C-H, Fu H, Lönnroth K, Chang Y-C, et al. Association of Obesity, Diabetes, and Risk of Tuberculosis: Two Population-Based Cohorts. Clin Infect Dis. 2018;66:699-705.

25. Yang W, Lu J, Weng J, Jia W, Ji L, Xiao J, et al. Prevalence of Diabetes among Men and Women in China. N Engl J Med. 2010;362:1090-101.

26. Schaible UE, Kaufmann SHE. Malnutrition and Infection: Complex Mechanisms and Global Impacts. PLoS Med. 2007;4:e115.

27. Cegielski JP, McMurray DN. The relationship between malnutrition and tuberculosis: evidence from studies in humans and experimental animals. Int $\mathrm{J}$ Tuberc Lung Dis. 2004;8:286-98.

28. Feng Y, Wang F, Pan H, Qiu S, Lü J, Wu L, et al. Obesity-associated gene FTO rs9939609 polymorphism in relation to the risk of tuberculosis. BMC Infect Dis. 2014;14:592.

29. Menzies NA, Wolf E, Connors D, Bellerose M, Sbarra AN, Cohen T, et al. Progression from latent infection to active disease in dynamic tuberculosis transmission models: a systematic review of the validity of modelling assumptions. Lancet Infect Dis. 2018;18:e228-38.

30. Saag LA, LaValley MP, Hochberg NS, Cegielski JP, Pleskunas JA, Linas BP, et al. Low body mass index and latent tuberculous infection: a systematic review and meta-analysis. Int J Tuberc Lung Dis. 2018;22:358-65.

31. Zhang H, Li X, Xin H, Li H, Li M, Lu W, et al. Association of Body Mass Index with the Tuberculosis Infection: a Population-based Study among 17796 Adults in Rural China. Sci Rep. 2017;7:41933.

\section{Figures}




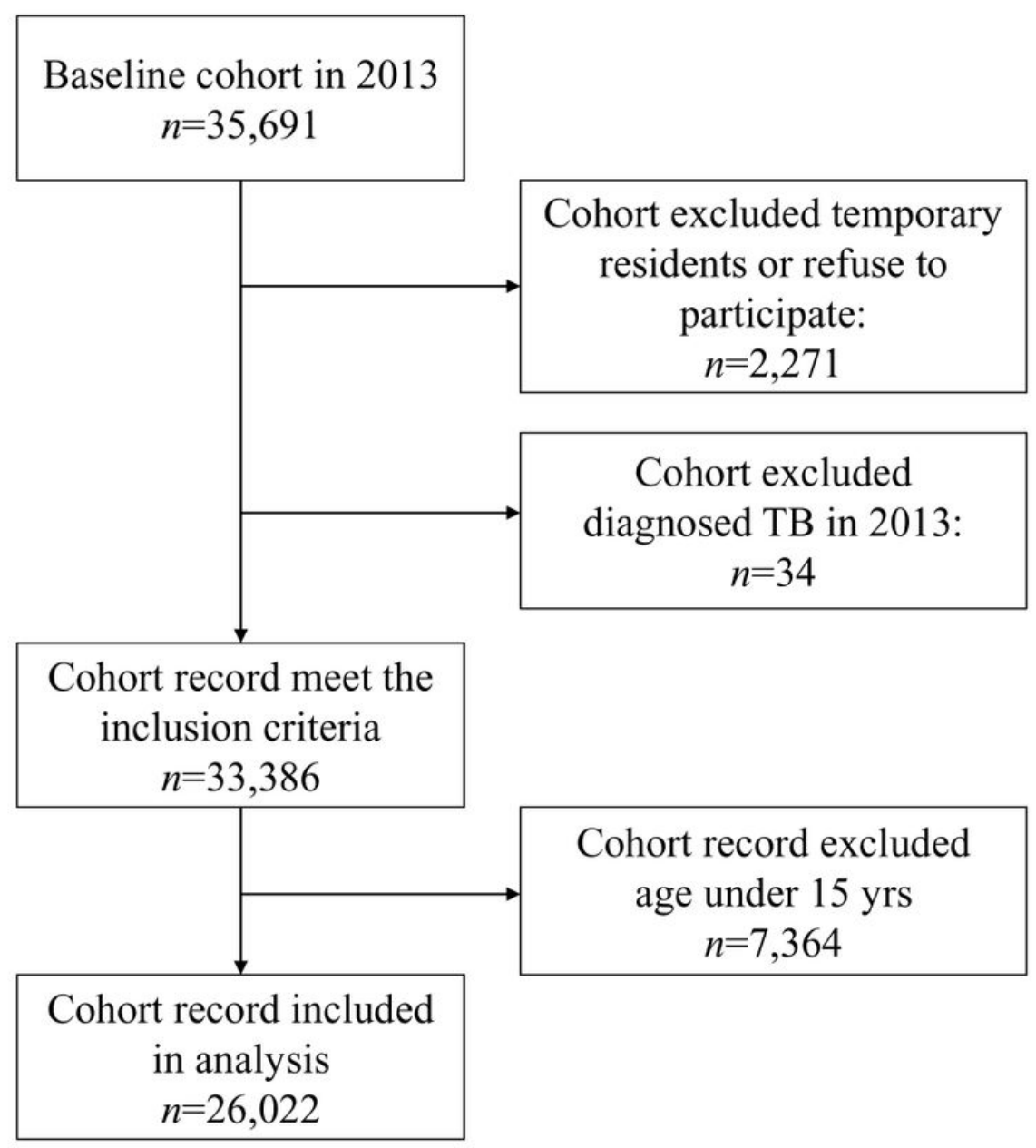

Figure 1

Diagram of the tuberculosis screening cohort between 2013 and 2015. Abbreviations: TB, tuberculosis 


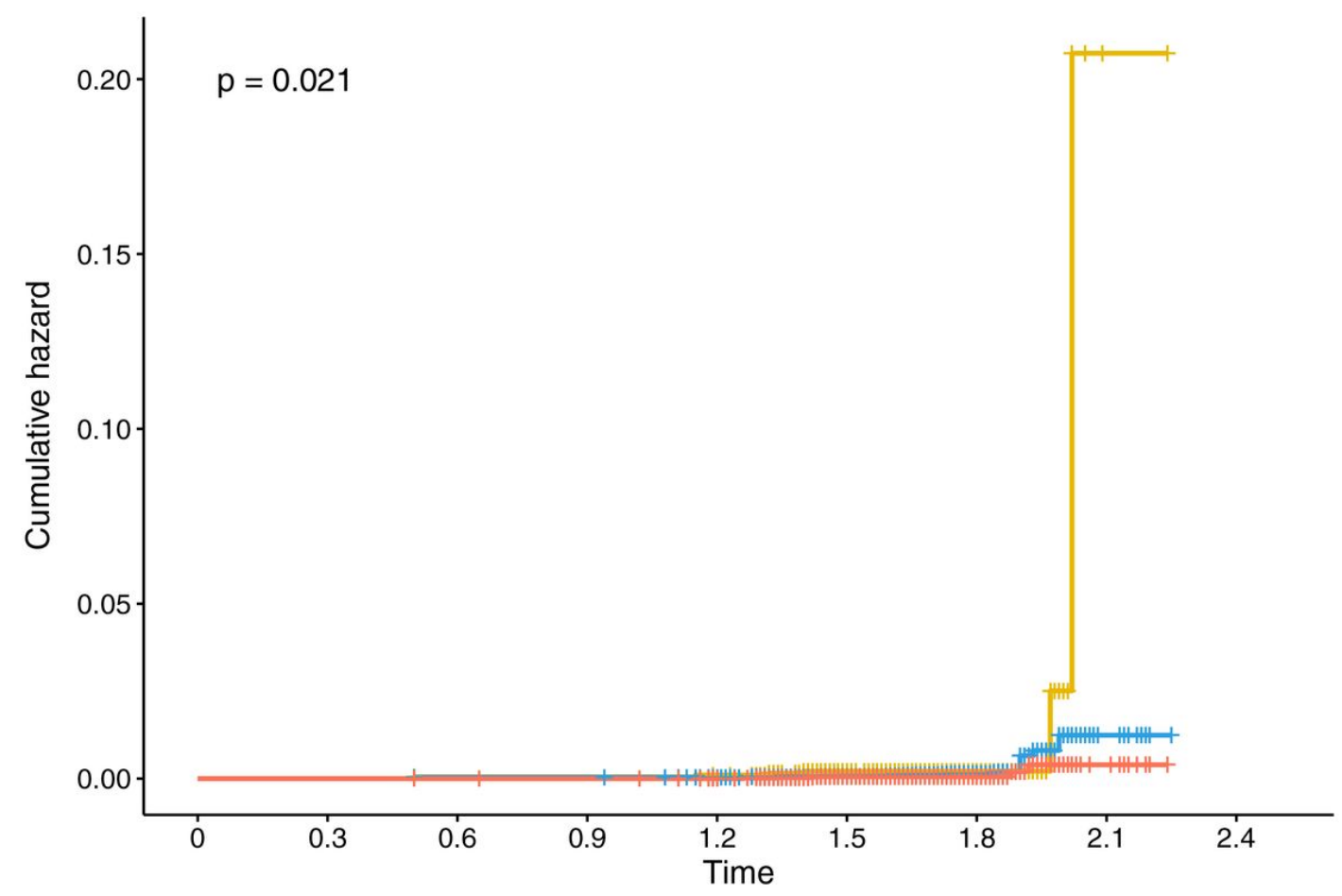

\section{Number at risk}

$\bar{\Sigma}=$\begin{tabular}{ccccccccc}
2042 & 2042 & 1870 & 1870 & 1868 & 1786 & 1581 & 1 & 0 \\
16009 & 16009 & 14794 & 14794 & 14779 & 14140 & 12587 & 12 & 0 \\
7971 & 7971 & 7461 & 7460 & 7452 & 7064 & 6124 & 9 & 0 \\
\hline 0 & 0.3 & 0.6 & 0.9 & $\begin{array}{c}1.2 \\
\text { Time }\end{array}$ & 1.5 & 1.8 & 2.1 & 2.4
\end{tabular}

$\mathrm{BMI}+$ Underweight + Normal + Overweight

Figure 2

Kaplan-Meier plot of cumulative hazard of tuberculosis by body mass index. The yellow line (Underweight) represents lower BMI (18.5<kg/m2), the blue line (Normal) represents the BMI between 18.5 and $24 \mathrm{~kg} / \mathrm{m} 2$, orange line (Overweight) represent BMI over $24 \mathrm{~kg} / \mathrm{m} 2$. The time to events or censor was defined by years. Abbreviations: TB, tuberculosis; BMI, body mass index 


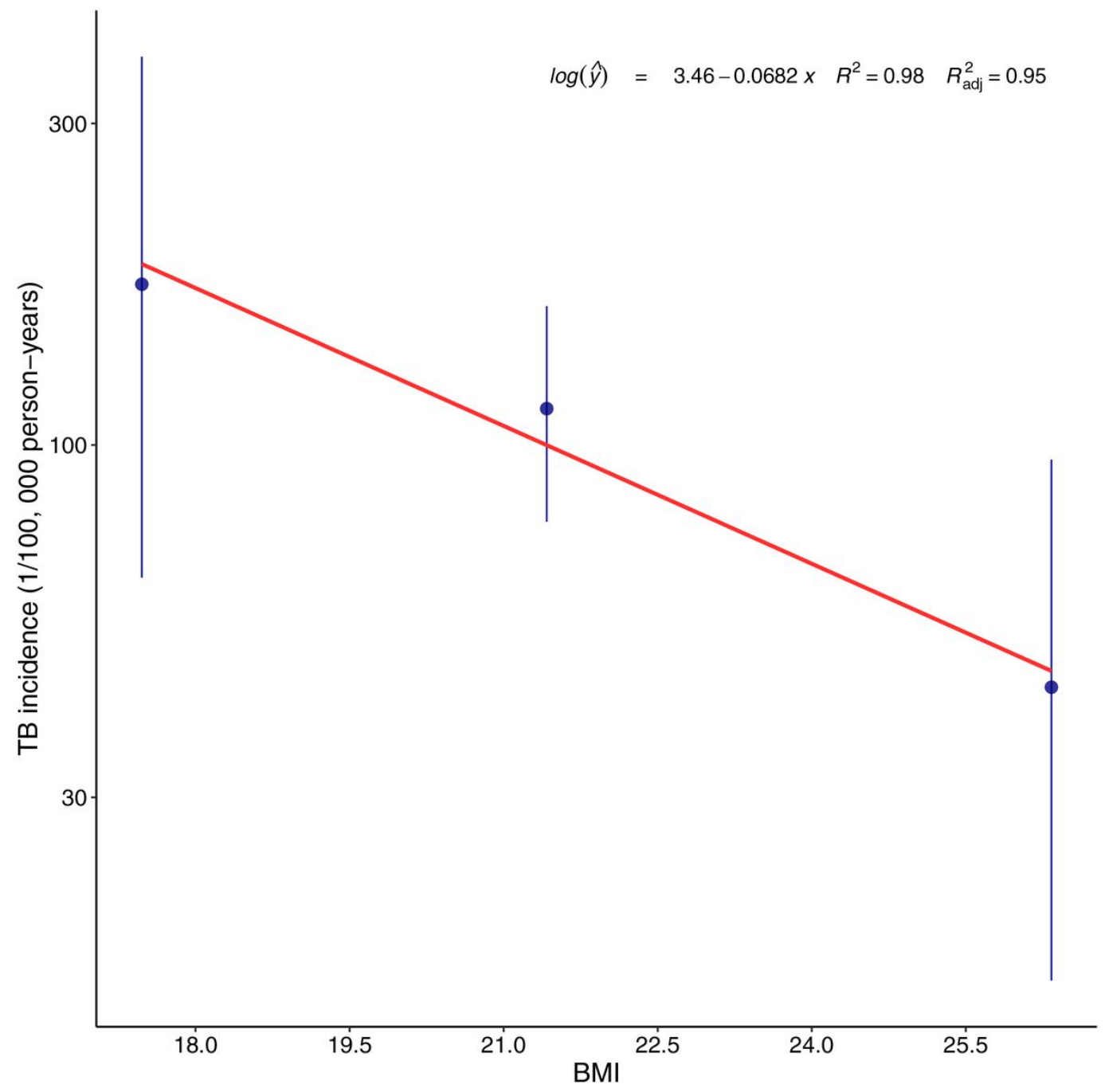

Figure 3

Dose-response relationship of the association between body mass index and the incidence of tuberculosis. TB incidence was transformed with denary logarithm, then regressed with a mean $\mathrm{BMI}$ of three categories. Blue points and ranges represent the $\mathrm{TB}$ incidence and $95 \% \mathrm{Cl}$ by the average $\mathrm{BMI}$ in three categories $(17.5 \mathrm{~kg} / \mathrm{m} 2,21.4 \mathrm{~kg} / \mathrm{m} 2$ and $26.3 \mathrm{~kg} / \mathrm{m} 2$ in underweight, normal and overweight groups respectively), the red line represents the log-linear dose-response relationship between BMI and TB incidence. Abbreviations: TB, tuberculosis; BMI, body mass index; $\mathrm{Cl}$, confidence interval; 


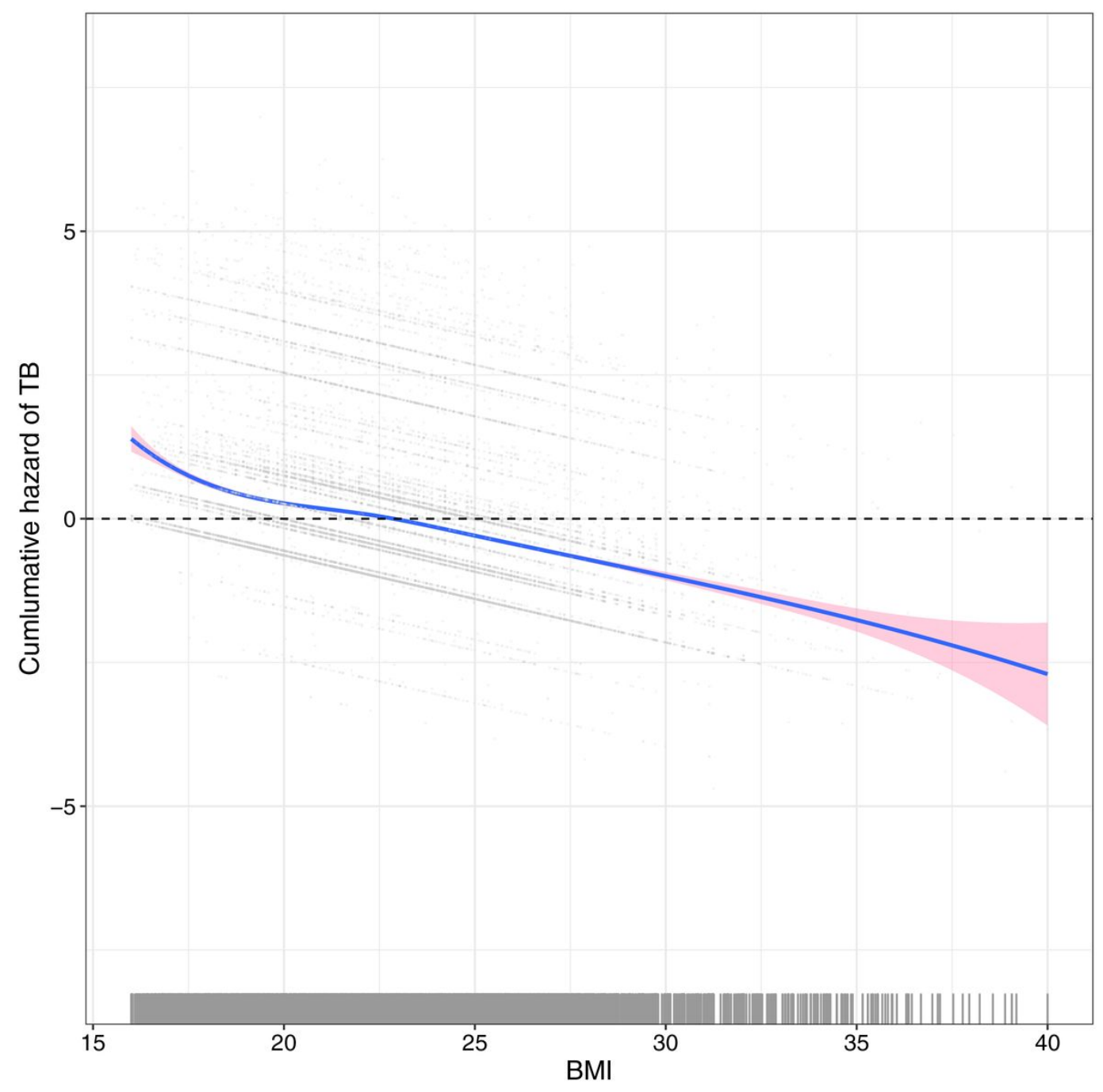

Figure 4

Dose-response curves for the body mass index and logarithmic cumulative hazard of tuberculosis in Cox proportional hazards model. The blue line and pink area represent the non-linear relationship of predicted log-linear cumulative hazard of TB and BMI using the restricted cubic spline regression, the spline knots of BMI were the quartiles $(25 \%, 50 \%$, and $75 \%)$ of the continuous variable. The Cox model adjusted for age, sex, ethnicity, marital status, previously treated TB, smoking status, alcohol use, known diabetes, and BMI. All variables were adjusted as categorical variables except continuous BMI. Abbreviations: TB, tuberculosis; $\mathrm{BMI}$, body mass index 

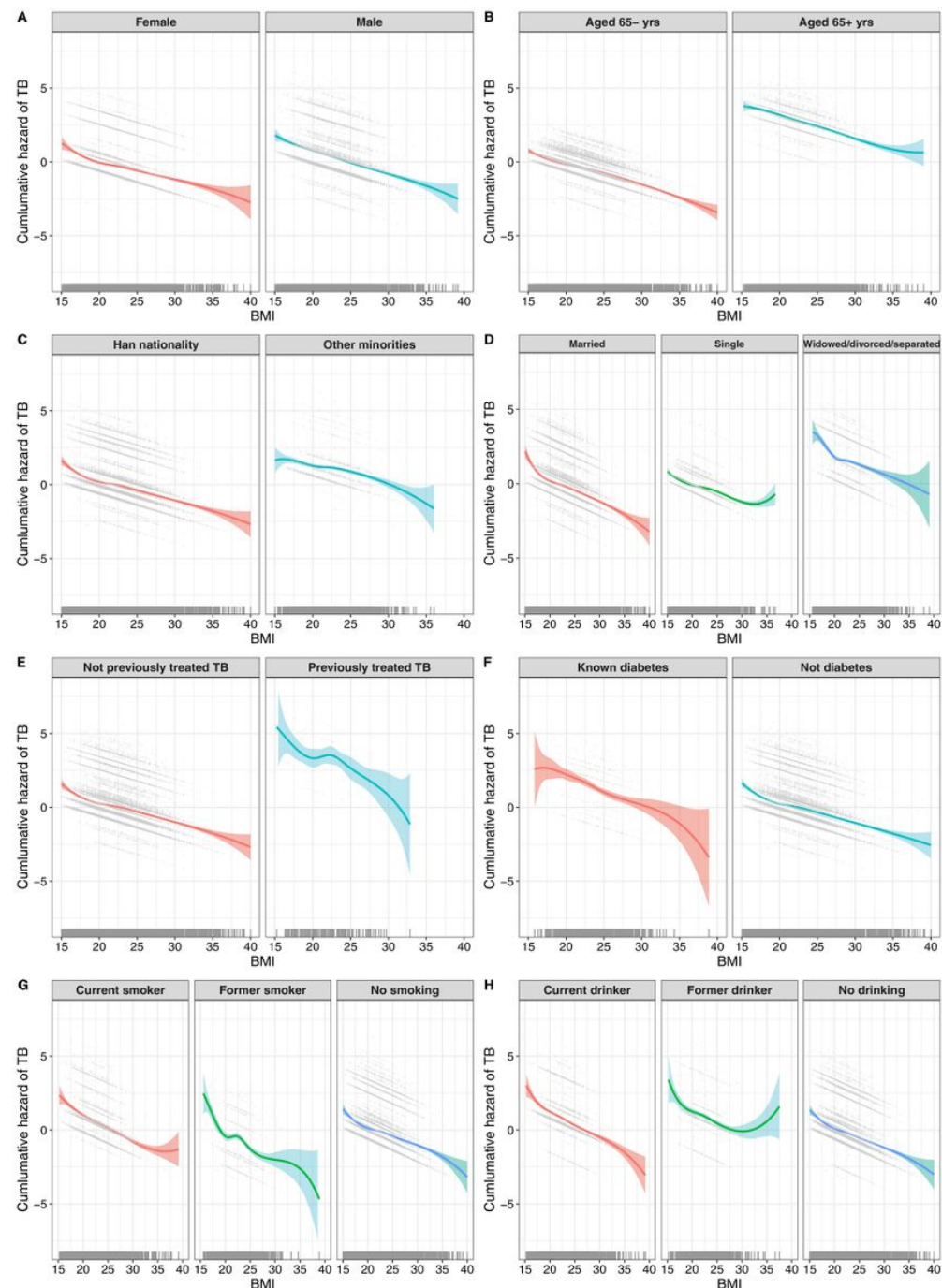

\section{Figure 5}

Dose-response curves for the body mass index and logarithmic cumulative hazard of tuberculosis in Cox proportional hazards model stratified by predictors. Panels represent different categories of predictors in the model and its non-linear relationship between predicted log-linear cumulative hazard of TB and BMI using the restricted cubic spline regression, the spline knots of BMI were the quartiles $(25 \%, 50 \%$, and $75 \%)$ of the continuous variable. The Cox proportional hazards model adjusted for age, sex, ethnicity, marital status, previously treated TB, smoking status, alcohol use, known diabetes, and BMI. All variables were adjusted as categorical variables except continuous BMI. (A)Sex, (B)Age, (C)Ethnicity, (D)Marital status; (E)Previously treated TB, (F)Known diabetes, (G)Smoking status, (H)Alcohol use Abbreviations: TB, tuberculosis; BMI, body mass index 